BMJ Open

Sport \&

Exercise

Medicine

\title{
Mental health and psychological well- being among professional rugby league players from the UK
}

\author{
Adam Robert Nicholls (D) , ${ }^{1}$ Daniel J Madigan (D) , ${ }^{2}$ Lucas R W Fairs, ${ }^{1}$ \\ Richard Bailey ${ }^{3}$
}

To cite: Nicholls AR,

Madigan DJ, Fairs LRW, et al. Mental health and psychological well-being among professional rugby league players from the UK. BMJ Open Sport \& Exercise Medicine 2020;6:e000711. doi:10.1136/ bmjsem-2019-000711

Accepted 6 February 2020

\section{Check for updates}

\section{(C) Author(s) (or their} employer(s)) 2020. Re-use permitted under CC BY-NC. No commercial re-use. See rights and permissions. Published by BMJ.

${ }^{1}$ Department of Sport, Health and Exercise Science, University of Hull, Hull, UK

${ }^{2}$ School of Sport, York Saint John University, York, UK

${ }^{3}$ International Council of Sport Science and Physical Education, Berlin, Germany

Correspondence to Dr Adam Robert Nicholls; A.Nicholls@hull.ac.uk

\section{ABSTRACT}

Objective This study aimed to measure depressive symptoms, anxiety symptoms and psychological wellbeing among a sample of Super League players, the highest tier of professional rugby league in the northern hemisphere.

Methods Between November 2018 and July 2019 a sample of 233 Super League players completed questionnaires that assessed depressive symptoms, anxiety symptoms and psychological well-being. Results Players exhibited normal (85.8\%), mild (11.6\%) or moderate/severe (2.6\%) depressive symptoms. Additionally, players reported normal (67.4\%), mild (18.9\%) or moderate/severe (13.7\%) anxiety symptoms. The mean psychological well-being score across the sample was 25.07 ( $\mathrm{SD}=4.34$ ). $35.2 \%$ of players scored below average for this construct.

Conclusion The prevalence of mild depressive symptoms, moderate/severe depressive symptoms and mild anxiety symptoms among Super League players preliminary appear higher than in the general population from the UK. Much more research is needed to understand mental health among elite athletes.

\section{INTRODUCTION}

Although elite athletes often epitomise the fit and healthy archetype, research over recent years suggests that they are still as likely, if not more likely, to be at risk of experiencing mental health symptoms. Several metaanalyses and/or reviews indicate that playing sport at the highest level is associated with significant mental health symptoms such as depressive and anxiety symptoms. ${ }^{1-5}$ It has been suggested that this may be due to the significant pressures of being an elite athlete, including high training loads, stress of competition and insufficient recovery. ${ }^{4}$ Mental health symptoms are a particular concern for elite athletes because they are associated with poor performance, ${ }^{6}$ which could then ultimately heighten symptoms of anxiety. ${ }^{7}$ Further, elite athletes with mental health symptoms may have a greater likelihood of getting injured, experiencing insomnia and having suicidal
What are the new findings?

- Mild depressive and anxiety symptoms preliminary appear more prevalent among Super League players than the general population from the UK.

- The prevalence of moderate/severe depressive symptoms appears higher among Super League players than the general population of the UK.

- Some previous studies that examined depressive and anxiety states as separate constructs may have underestimated the number of elite athletes with anxiety symptoms.

- Over a third of Super League players scored below average for psychological well-being.

thoughts in comparison to elite athletes with no mental health symptoms. ${ }^{1}$

The prevalence of depressive and anxiety symptoms among elite athletes is equivocal. For example, a meta-analysis, containing nine studies that assessed a combined score of anxiety and depressive symptoms, revealed that $33.6 \%$ of athletes reported symptoms of anxiety/depression. ${ }^{3}$ Another scoping review reported that elite athletes reported depressive symptoms between $4 \%$ and $68 \%$ and anxiety between $6 \%$ and $14.6 \% .^{1}$ Another meta-analysis, ${ }^{2}$ which compared the prevalence of depressive and anxiety symptoms among elite athletes and the general population, reported that neither depressive symptoms nor anxiety symptoms are more common among elite athletes than the general population.

There are two studies that have examined similar athletes as the present study. First, Foskett and Longstaff ${ }^{8}$ found that $47.8 \%$ of elite athletes from the UK reported symptoms of anxiety/depression. Second, Du Preez et at found that $12.6 \%$ of professional rugby league players from Australia's National Rugby League (NRL) experienced depressive symptoms during preseason, whereas only $10.1 \%$ reported depressive symptoms during the season. Moreover, $14.1 \%$ of these NRL 
players reported anxiety symptoms during preseason, compared with $10.1 \%$ of players during the season.

These equivocal findings from meta-analyses, scoping reviews and systematic reviews regarding depressive and anxiety symptoms ${ }^{1-5}$ may be due to differences in the content of questionnaires used in these studies, which have not been validated for elite athletes. Some studies $^{9}{ }^{10}$ assessed anxiety using the General Anxiety Disorder (GAD) scale, ${ }^{11}$ whereas another study ${ }^{8}$ used the General Health Questionnaire (GHQ-12) ${ }^{12}$ The GAD and the GHQ-12 are not as accurate at screening for anxiety and/or depressive symptoms ${ }^{13} 14$ as the Hospital Anxiety Depression Scale (HADS).$^{15}$ As such, it could be argued that assessing anxiety and depression using the HADS ${ }^{15}$ would more accurately measure the prevalence of anxiety and depressive symptoms among elite athletes. With this in mind, scholars have stated that more thorough and reliable mental health epidemiology data are needed among elite athletes. ${ }^{9}$ Using a more sensitive questionnaire, such as the HADS, may provide such information among elite athletes, in order to make comparisons between elite athletes and the general population.

Breeman et $a l^{16}$ conducted a large-scale study with 6189 non-clinical participants to assess the prevalence of anxiety and depressive symptoms among the general population from the UK. Scores on the depressive and anxiety subscale of $\mathrm{HADS}^{15}$ range from 0 to 21 . A score ranging from 0 to 7 is 'normal,' which means the individual is not experiencing clinical symptoms of either depression or anxiety. A score of 8-10 on either the depressive or anxiety subscale indicates that a person is experiencing mild symptoms of either mood state. Such a score is suggestive of a mood disorder. A score of 11-21 was classified as moderate to severe anxiety or depression and represents a definitive case of clinical anxiety or depressive disorders. ${ }^{16}$ The prevalence of mild and moderate/severe depressive symptoms among those aged 25-29years old was $6.4 \%$ and $4.6 \%$, respectively. Furthermore, the prevalence of depressive symptoms among those aged $30-34$ years old was $7.0 \%$ (mild symptoms) and $4.9 \%$ (moderate/severe). The prevalence rates of mild anxiety among the general population aged 25-29 years old was $13.8 \%$ and $12.7 \%$ for $30-34$ years old. Moreover, $13.8 \%$ of those aged 25-29years old and $15.6 \%$ of those aged 30-34years old experienced moderate to severe symptoms of anxiety.

A notable omission in the literature are psychological well-being epidemiological studies among elite athletes. Reardon and colleagues ${ }^{1}$ argued that it is important to consider psychological well-being, as well as mental health symptoms among elite athletes. Psychological well-being is associated with athletes responding positively to goals that are difficult to achieve by attempting alternative strategies, using coping strategies that are associated with superior sporting performance, and perceiving stressful sporting events as challenging. ${ }^{17}$ Psychological well-being is therefore desirable among athletes, so understanding the levels of psychological well-being among elite athletes is important in order to ascertain levels of happiness and pleasure (hedonic well-being) and the extent to which a person is functioning fully (eudaimonic well-being). ${ }^{18}$

Following calls for more thorough mental health epidemiology research among elite athletes, ${ }^{9}$ the aim of the present study was to examine the prevalence of depressive symptoms, anxiety symptoms and psychological well-being among a sample of UK Super League players and to compare these findings with previous research featuring other elite athletes and the general population from the UK. Reporting the prevalence of mental health symptoms among Super League players will reveal whether this specific group of athletes is at risk of developing mental health symptoms. This could then provide a rationale for the management of players and optimising training environments across different clubs in order to maximise mental health among Super League players. $^{14}$

\section{MATERIALS AND METHODS}

\section{Study design and participants}

The Super League is the highest tier of professional rugby league in the northern hemisphere. Twelve professional clubs participate in the Super League, and each club employs about 30 players in their first team squad. Eleven Super League clubs participated in this study. Following ethical approval, player welfare managers at each club explained the study and invited players to participate, via email, and sent one reminder email every 2 months. Up to three reminder emails were sent to a player if they had not participated in the study. Data for this cross-sectional study were collected between November 2018 and July 2019, which is during preseason and competitive season. Participants completed the questionnaires anonymously, via an online platform (Qualtrics).

A total of 233 male professional Super League players participated in the present study. Participants' mean age was 24.35 years $(\mathrm{SD}=5.20$; range $=16-38$ years $)$. Participants had been professional players for 7.03 years $(\mathrm{SD}=5.07)$ and had played in the Super League for 4.74 years $(\mathrm{SD}=4.77)$.

\section{Measures}

Depression and anxiety

$\mathrm{HADS}^{15}$ is a 14-item self-report instrument to screen for clinical depressive and anxiety symptoms in a nonpsychiatric hospital setting. The two subscales (ie, depression and anxiety) of HADS each contain seven statements, which are answered on a Likert-type scale ranging between 0 and 3 . Although the $\operatorname{HADS}^{15}$ has not been validated with samples of elite athletes, Cronbach's alpha in the present study were acceptable for both depression (0.71) and anxiety (0.81).

Psychological well-being

The Short Warwick Edinburgh Mental Well-being Scale $(\text { SWEMWBS })^{19}$ assessed psychological well-being. The 
SWEMWBS instructs participants to indicate the level of occurrence of the presented statements over a 2-week period on a five-point Likert scale, anchored at 1 'none of the time' and 5 'all of the time'. The SWEMWBS has not been validated for use with elite athletes, but had acceptable levels of reliability with our sample, as the Cronbach alpha in the present study was 0.84 .

\section{Patient and public involvement}

No patients were involved in this study because the purpose was to assess the prevalence of depression, anxiety and well-being among Super League players. Each participant received a summary of their findings. Player welfare managers, employed by each Super League club, invited participants to discuss their findings.

\section{Preliminary analyses}

First, we inspected the data for missing values. Very few item responses were missing $(i=5)$, so missing responses were replaced with the mean of the item responses of the corresponding scale. ${ }^{20}{ }^{21}$ Next, we computed Cronbach's alphas for our variables, which were all satisfactory.

\section{RESULTS}

Two hundred players reported a normal score for depressive symptoms, whereas $27(11.6 \%)$ players reported mild depressive symptoms, and $6(2.6 \%)$ suffered from moderate/severe depressive symptoms. In regard to anxiety symptoms, 157 (67.4\%) reported normal scores, $44(18.9 \%)$ players reported mild anxiety symptoms and $32(13.7 \%)$ players exhibited moderate/severe anxiety symptoms. The mean psychological well-being score across the sample was $25.07(\mathrm{SD}=4.34)$. More specifically, $29(12.4 \%)$ players scored above average for psychological well-being, $122(52.4 \%)$ had an average score and 82 $(35.2 \%)$ score below average for psychological well-being (table 1).

\section{DISCUSSION}

The purpose of this epidemiological study was to examine the prevalence of depressive symptoms, anxiety symptoms and psychological well-being among Super League players. Overall, $14.2 \%$ of the players displayed some depressive symptoms and $32.6 \%$ reported symptoms of anxiety beyond a normal level. With regard to psychological well-being, the mean score was 25.07 , with $35.2 \%$ of the sample scoring below average.

\section{Depressive symptoms}

The percentage of Super League players displaying symptoms of depression is somewhat comparable with $\mathrm{Du}$ Preez and colleagues, ${ }^{9}$ but substantially lower than other studies featuring athletes from different individual and team sports. ${ }^{8} 10$ Research comparing the prevalence of depressive symptoms among individual and team sport athletes revealed that individual sport athletes are more likely to report depressive symptoms than team sport athletes. ${ }^{6}$ A possible reason for this is a protective mechanism of being involved in a team sport. The present study, and the research by Du Preez et $a l,{ }^{9}$ included a sample of professional rugby league players. Other two studies ${ }^{810}$ included athletes from different sports including individual sports. Consequently, these differences could be partly explained by the recruitment and analysis of different athlete groups from differing sports.

With regard to comparing the prevalence of depressive symptoms among Super League players with the general population from the UK, comparisons must remain conjectural as all participants in the Breeman et al study ${ }^{16}$ were aged 25 years or older. The youngest players in this study were aged 16 years old. However, Super League players scored higher on both mild and moderate/severe symptoms than men aged 25-29 and 30-34years old from the general population in the UK. ${ }^{16}$ Even though the prevalence of mild and moderate/severe depressive symptoms are higher among Super League players than the general population, the prevalence rates within professional rugby league might be even higher than those reported in this study. There is evidence to suggest that depression can lead to decreased performance and ultimately athletes exiting sport. ${ }^{16}$ It would be interesting to assess the numbers of Super League players who have recently exited the Super League to fully examine the prevalence of depressive symptoms among this group of elite athletes fully.

\section{Anxiety symptoms}

The prevalence of mild and moderate/severe symptoms of anxiety appears to be higher in the present study than in some reports. ${ }^{910}$ This could be because HADS $^{6}$ is more sensitive at detecting anxiety symptoms than both the $\mathrm{GAD}^{4}$ and the GHQ- $12 .^{5}$ As such, previous studies ${ }^{910}$ may have underestimated the prevalence of anxiety symptoms among elite athletes.

Table 1 Scores for depression, anxiety and psychological well-being

\begin{tabular}{|c|c|c|c|c|}
\hline & Mean (SD) & Players, n (\%) & & \\
\hline & & Normal (score 0-7) & Mild (score 8-10) & Moderate/severe (score $\geq 11$ ) \\
\hline Depression & $3.93(2.98)$ & $200(85.8 \%)$ & $27(11.6 \%)$ & $6(2.6 \%)$ \\
\hline \multirow[t]{2}{*}{ Anxiety } & $6.42(3.59)$ & $157(67.4 \%)$ & 44 (18.9\%) & 32 (13.7\%) \\
\hline & & Above average (score $\geq 30$ ) & Average (score 24-29) & Below average (score $\leq 23$ ) \\
\hline Psychological well-being & $25.07(4.34)$ & 29 (12.4\%) & $122(52.4 \%)$ & $82(35.2 \%)$ \\
\hline
\end{tabular}


The prevalence rates of mild anxiety symptoms among Super League players appear higher than the general population aged 25-29(13.8\%) and 30-34years old $(12.7 \%)$. However, the prevalence rates for moderate to severe symptoms among these players and those aged 25-29 (13.8\%) and 30-34years old (15.6\%) are quite similar, which agrees with Rice et al. ${ }^{2}$ It would be interesting to examine the impact of anxiety symptoms among players who have exited the sport because anxiety is linked to poorer skill execution. ${ }^{17}$ Highly anxious elite athletes in team sports, such as rugby league players, may leave the sport because their contracts are not renewed because of declining performance. This could be examined in future studies with elite athletes, by monitoring anxiety rates over a prolonged period and monitoring exiting rates that are self-determined or imposed on the athlete.

\section{Psychological well-being}

This is the first study to examine psychological well-being in elite rugby league players. The mean psychological well-being score in the present study (25.07) is slightly higher than the mean score for men in the general population in the UK (23.7). ${ }^{22}$ Worryingly, $35.2 \%$ of Super League players score below average, which may be indicative of them not coping well, being unhappy and not functioning fully. In order to address this concern, well-being interventions that enhance the ability to cope and support happiness are required. In their consensus statement on mental health among athletes, Reardon and colleagues ${ }^{1}$ stated that scholars should monitor the impact of interventions on both mental health symptoms and well-being. The findings of the present study endorse this sentiment because it is important that psychological interventions for elite athletes not only reduce symptoms of anxiety and depression but also enhance psychological well-being.

\section{Limitations}

Comparing the prevalence of depression and anxiety to other studies featuring these constructs among elite athletes $^{8-10}$ may not be very meaningful because of the range of different measures used to assess these constructs. The comparisons made between previous research and this study are done with this caveat in mind. A limitation of this study is that findings might not be fully representative of all Super League players because $30 \%$ of players chose not to participate in this study. Some players might have chosen not to participate because they have experienced no or very mild symptoms of anxiety or depression, whereas others might not have wanted to take part because of the stigma associated with mental health among elite athletes. ${ }^{23}$ Furthermore, data in this study are cross-sectional and collected at one time point. The timing of administering mental health questionnaires, however, does not appear to significantly influence scores for depressive or anxiety symptoms. ${ }^{6}$ Indeed, Du Preez et at found that that depressive and anxiety symptoms remained relatively stable among players who completed questionnaires during preseason and in-season.

\section{CONCLUSIONS}

The percentage of Super League players with mild depressive symptoms preliminary appear higher than in the general population of the UK. The prevalence of anxiety symptoms among Super League players appears higher than some studies with other elite athletes. ${ }^{9} 10$ Furthermore, the prevalence of mild anxiety symptoms among Super League players is higher than the general population. Due to the issues raised around the measurement effectiveness of mental health symptoms, further research is required to accurately detect depressive and anxiety symptoms among elite athletes. Regular screening should be offered for all elite athletes, so that they can receive help if and when they need it. The effectiveness of interventions designed to reduce mental health symptoms and enhance psychological well-being is warranted, as is a more detailed examination of the factors that underpin mental health and well-being.

Acknowledgements We are grateful to the RFL, Emma Rosewarne and the player welfare managers at each club.

Contributors ARN conceptualised the study, managed data collection and wrote the paper. DM analysed the data and contributed to the write up. LRWF collected data and contributed to the write up. RB contributed to the write up.

Funding The authors have not declared a specific grant for this research from any funding agency in the public, commercial or not-for-profit sectors.

\section{Competing interests None declared.}

Patient consent for publication Not required.

Provenance and peer review Not commissioned; externally peer reviewed.

Data availability statement Data are available on reasonable request. Data are available on request from the corresponding author.

Open access This is an open access article distributed in accordance with the Creative Commons Attribution Non Commercial (CC BY-NC 4.0) license, which permits others to distribute, remix, adapt, build upon this work non-commercially, and license their derivative works on different terms, provided the original work is properly cited, appropriate credit is given, any changes made indicated, and the use is non-commercial. See: http://creativecommons.org/licenses/by-nc/4.0/.

ORCID iDs

Adam Robert Nicholls http://orcid.org/0000-0002-8883-5445

Daniel J Madigan http://orcid.org/0000-0002-9937-1818

\section{REFERENCES}

1 Reardon CL, Hainline B, Aron CM, et al. Mental health in elite athletes: international Olympic Committee consensus statement (2019). Br J Sports Med 2019;53:667-99.

2 Rice SM, Gwyther K, Santesteban-Echarri O, et al. Determinants of anxiety in elite athletes: a systematic review and meta-analysis. $\mathrm{Br} J$ Sports Med 2019;53:722-30.

3 Gouttebarge V, Castaldelli-Maia JM, Gorczynski P, et al. Occurrence of mental health symptoms and disorders in current and former elite athletes: a systematic review and meta-analysis. Br J Sports Med 2019;53:700-6.

4 Kuettel A, Larsen $\mathrm{CH}$. Risk and protective factors for mental health in elite athletes: a scoping review. Int Rev Sport Exerc Psych 2019.

5 Rice SM, Purcell R, De Silva S, et al. The mental health of elite athletes: a narrative systematic review. Sports Med 2016;46:1333-53.

6 Wolanin A, Gross M, Hong E. Depression in athletes: prevalence and risk factors. Curr Sports Med Rep 2015;14:56-60.

7 Nicholls AR, Taylor NJ, Carroll S, et al. The development of a new sport-specific classification of coping and a meta-analysis of the 
relationship between different coping strategies and Moderators on sporting outcomes. Front Psychol 2016;7:1674.

8 Foskett RL, Longstaff F. The mental health of elite athletes in the United Kingdom. J Sci Med Sport 2018;21:765-70.

9 Du Preez EJ, Graham KS, Gan TY, et al. Depression, anxiety, and alcohol use in elite rugby league players over a competitive season. Clin J Sport Med 2017;27:530-5.

10 Gulliver A, Griffiths KM, Mackinnon A, et al. The mental health of Australian elite athletes. J Sci Med Sport 2015;18:255-61.

11 Spitzer RL, Kroenke K, Williams JBW, et al. A brief measure for assessing generalized anxiety disorder: the GAD-7. Arch Intern Med 2006;166:1092-7.

12 Goldberg DP, Gater R, Sartorius N, et al. The validity of two versions of the GHQ in the WHO study of mental illness in general health care. Psychol Med 1997;27:191-7.

13 Baker AM, Holbrook JT, Yohannes AM, et al. Test performance characteristics of the AIR, GAD-7 and HADS anxiety screening questionnaires for anxiety in chronic obstructive pulmonary disease. Ann Am Thorac Soc 2018;15:926-34.

14 Hahn D, Reuter K, Härter M. Screening for affective and anxiety disorders in medical patients - comparison of HADS, GHQ-12 and Brief-PHQ. Psychosoc Med 2006;3:Doc09.

15 Zigmond AS, Snaith RP. The hospital anxiety and depression scale. Acta Psychiatr Scand 1983;67:361-70.
16 Breeman S, Cotton S, Fielding S, et al. Normative data for the hospital anxiety and depression scale. Qual Life Res 2015;24:391-8.

17 Nicholls AR, Levy AR, Carson F, et al. The applicability of selfregulation theories in sport: goal adjustment capacities, stress appraisals, coping, and well-being among athletes. Psychol Sport Exerc 2016;27:47-55.

18 Ryan RM, Deci EL. On Happiness and human potentials: a review of research on hedonic and Eudaimonic well-being. Annu Rev Psychol $2001 ; 52: 141-66$

19 Tennant R, Hiller L, Fishwick R, Platt S, et al. The Warwick-Edinburgh mental well-being scale (WEMWBS): development and UK validation. Health Qual Life Outcomes 2007;5:63-13.

20 Graham JW, Cumsille PE, Elek-Fisk E. Methods for handling missing data. In: Schinka JA, Velicer WF, eds. Handbook of psychology: research methods in psychology. New York: Wiley, 2003: 87-114.

21 Bell ML, Fairclough DL, Fiero MH, et al. Handling missing items in the hospital anxiety and depression scale (HADS): a simulation study. BMC Res Notes 2016;9:479.

$22 \mathrm{Ng}$ Fat L, Scholes S, Boniface S, et al. Evaluating and establishing national norms for mental wellbeing using the short WarwickEdinburgh mental well-being scale (SWEMWBS): findings from the health survey for England. Qual Life Res 2017;26:1129-44.

23 Gulliver A, Griffiths KM, Christensen H. Barriers and facilitators to mental health help-seeking for young elite athletes: a qualitative study. BMC Psychiatry 2012;12:157. 